\title{
RICOSTRUZIONE DI PROFILI DI CONTENUTO D'ACQUA IN UN SUOLO STRUTTURATO SOTTOPOSTO A LAVORAZIONI
}

\author{
Paolo Damiani, Guido Ciollaro, Antonio Coppola
}

\section{INTRODUZIONE}

\section{Introduction}

Il movimento dell' acqua nel suolo avviene secondo meccanismi che variano in dipendenza della distribuzione e della configurazione dei vuoti nel sistema poroso. Nei mezzi porosi strutturati, il moto viene frequentemente descritto come combinazione del flusso matriciale, che ha luogo nei pori presenti fra le particelle di suolo, e del flusso nei macropori, più rapido rispetto al primo, che impegna gli spazi originati dalla struttura e le eventuali fratture.

A tale proposito è bene ricordare che la struttura del suolo può essere descritta con diverse definizioni: "l'aggregazione di particelle primarie in unità secondarie di suolo" [11]; "la composizione di particelle primarie e della sostanza organica in aggregati ed il corrispondente sistema di porosità fra gli aggregati“* [5]. In pratica si può considerare strutturato un suolo in cui sono presenti porosità fra aggregati, fratture o comunque macropori al cui interno si instaura un flusso preferenziale.

Come per la struttura, anche per il flusso preferenziale esistono diverse definizioni: "il flusso preferenziale è quel flusso di acqua che avviene nei vuoti continui aventi un diametro che varia in un campo compreso tra 0,03 e $30 \mathrm{~mm}$ " [1]. In generale, il flusso preferenziale di acqua si instaura in volumi limitati di suolo, anche nel caso di apporti uniformemente distribuiti sulla superficie [6].

In dipendenza della geometria e dimensione dei macropori, e dei flussi che conseguentemente vi si instaurano, l'equazione di Richards, generalmente utilizzata per descrivere il moto della fase liquida nella matrice del suolo, può risultare più o meno idonea $\mathrm{a}$

\section{Memoria presentata 08.02.2007; accettata 07.03.2008}

Damiani Paolo, Ciollaro Guido, Università degli Studi di Bari Dipartimento PRO.GE.SA - via Amendola 165/A - 70126 Bari.

Coppola ANTONIO, Università degli Studi della Basilicata - Dipartimento per la Gestione del Territorio Agro-Forestale (DITEC) - Sezione Idraulica - via dell'Ateneo Lucano - 85100 Potenza, acoppola@ unibas.it rappresentare il moto nei macropori.

Nel caso di fratture, in cui si instaurano flussi generalmente consistenti (con valori elevati del numero di Reynolds) il moto della fase liquida nel dominio strutturale andrebbe, a rigore, descritto utilizzando un approccio del tipo dell'onda cinematica o l'equazione di Hagen-Poiseuille, prevedendo poi un meccanismo di scambio fra il dominio strutturale e quello matriciale.

Un approccio alternativo può essere quello di considerare ancora il suolo strutturato come un mezzo continuo in cui la complessa eterogeneità può essere descritta con una distribuzione bimodale della porosità, introducendo una partizione fra componenti strutturale e matriciale del sistema poroso ed in cui il flusso preferenziale associato alla macroporosità possa ancora essere descritto con l'equazione di Richards. In presenza di fratture, tuttavia, l'applicabilità di questo approccio può risultare limitata, specie per i valori del contenuto d'acqua prossimi alla saturazione.

D'altra parte, la struttura, e quindi lo stato di aggregazione del mezzo poroso, possono risultare alterate per effetto di azioni esterne. Le lavorazioni del terreno, per esempio, modificano ciclicamente lo stato di aggregazione del suolo in superficie, con la struttura che tende a rigenerarsi con l'alternanza dei processi di inumidimento ed essiccamento del suolo. In effetti, le lavorazioni producono una modifica del profilo di suolo, inducendo la formazione di uno strato superficiale, il cui comportamento idrologico può risultare anche sensibilmente diverso da quello del suolo sottostante rimasto inalterato.

Obiettivo dello studio presentato in questo lavoro è stato quindi quello di valutare l'effetto delle lavorazioni sui processi idrologici che si manifestano in un suolo strutturato, avvalendosi di misure del contenuto idrico e dei potenziali lungo il profilo, oltre che delle precipitazioni.

Per il suolo esaminato, viene adottata una formulazione dell'equazione di Richards che tiene conto della eterogeneità del sistema poroso attraverso una rappresentazione bimodale delle proprietà idrauliche. 


\section{APPROCCIO TEORICO}

\section{Theoretical approach}

A scala locale il moto dell'acqua in un mezzo poroso non saturo è adeguatamente descritto dalla classica equazione di Richards:

$$
C \frac{\partial h}{\partial l}=\frac{\partial}{\partial z}\left(k(h) \frac{\partial h}{\partial z}-k(h)\right)
$$

Nell'equazione (1), qui considerata nella sua forma unidimensionale con flusso verticale, $C=d \theta / d h$ è la capacità capillare, $\theta$ è il contenuto idrico volumetrico, $h$ è il potenziale idraulico, negativo in un suolo non saturo, $t$ è il tempo, $k$ è la conducibilità idraulica e $z$ è la coordinata verticale, positiva verso il basso. L'equazione è risolvibile numericamente una volta note le condizioni al contorno ed iniziali e le proprietà idrauliche del suolo. Per l'utilizzo in un modello di simulazione, le proprietà idrauliche sono descritte da espressioni analitiche $\theta(h)$ (curve di ritenzione) e $k$ $(\theta)$ (curve di conducibiltà) in cui compaiono parametri che caratterizzano il suolo in esame [12]. Queste funzioni, generalmente, sono riferibili ad una distribuzione unimodale delle dimensioni dei pori. L'espressione di van Genuchten per un suolo unimodale si scrive:

$$
S_{r}=\frac{\theta-\theta_{r}}{\theta,-\theta}=\left[\frac{1}{1+\left(a^{n c} h\right)^{r}}\right]^{n}
$$

in cui $S_{e}$ è il grado di saturazione, $\theta_{\mathrm{s}}$ e $\theta_{\mathrm{r}}$ sono rispettivamente il contenuto d'acqua alla saturazione e quello residuo, $\alpha^{V G}, n$ e $m$ sono parametri di forma.

In suoli strutturati, il comportamento dei pori fra gli aggregati modifica significativamente la pendenza della curva di ritenzione in prossimità della saturazione. Questa condizione può essere descritta numericamente considerando una porosità composita in cui si assume che lo spazio tra i pori compreso fra il contenuto d'acqua residuo $\theta_{r}$ e quello alla saturazione $\theta_{s}$ segua due diverse distribuzioni di drenaggio, ciascuna occupante una frazione $\beta_{i}$ dello spazio dei pori.

La curva di ritenzione introdotta da Ross e Smettem [9] per un sistema poroso bimodale è espressa dalla relazione:

$$
\begin{gathered}
S_{c}=\beta_{1}\left(1+\alpha^{e s} h\right) \exp \left(-\alpha^{e s} h\right)+\beta_{2}\left[\frac{1}{1+\left(\alpha^{n i i} h\right)^{\gamma}}\right] \\
0<\beta_{i}<1 \text { < } \sum \beta_{i}=1 \quad i=1,2
\end{gathered}
$$

dove una semplice funzione ad un parametro $\left(\alpha^{R S}\right)$ tiene conto della macroporosità, mentre la curva di van Genuchten descrive la ritenzione idrica della matrice. $\beta_{i}$ è la porzione della porosità totale da attribuire alla curva iesima. Nota la saturazione, $S_{e}$, si considera una funzione di distribuzione cumulata della dimensione dei pori [4].

L'espressione di Mualem per calcolare la conduci- bilità idraulica relativa, $k_{r}$, [8] è espressa da:

$$
\begin{aligned}
& k_{,}\left(s_{,}\right)=\frac{k\left(s_{j}\right)}{k_{0}}= \\
& =S^{+}\left[\int \frac{1}{h(S)} d S / \int \frac{1}{h\left(S_{e}\right)} d S\right]^{2}
\end{aligned}
$$

in cui $k_{0}$ è la conducibilità idraulica alla saturazione $\mathrm{e}$ $\tau$ è un parametro connesso alla tortuosità dei percorsi di flusso.

Nel caso di distribuzione bimodale la (4a) diventa:

$$
\begin{aligned}
& k,(s)=\frac{k(s)}{k_{n}}=
\end{aligned}
$$

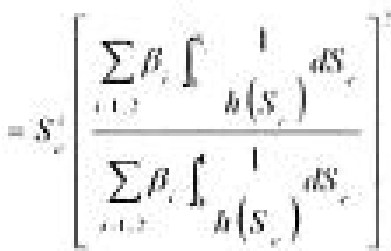

Le equazioni (4) consentono di ottenere una stima della funzione di conducibilità idraulica una volta noto $k_{0}$, attraverso i parametri che compaiono nelle equazioni (2) e (3), stimati adattando alle equazioni i dati misurati di ritenzione attraverso una procedura di ottimizzazione non-lineare dei minimi quadrati.

Se il flusso preferenziale è dovuto principalmente ai pori fra gli aggregati (con dimensione confrontabile a quella dei pori fra le particelle) può essere ragionevole assumere che la (1) sia ancora valida per descrivere con buona approssimazione il flusso nei macropori [7].

\section{MATERIALI E METODI}

\section{Materials and methods}

\subsection{IL CAMPO SPERIMENTALE}

\subsection{The Experimental Site}

L'area sperimentale (figura 1) rappresentativa dell'ambiente collinare meridionale, ricade nel bacino dell'Agri, sottobacino del Sauro, in tenimento di Guardia Perticara, Potenza, ad una quota di $720 \mathrm{~m}$ s.l.m., con una temperatura annua media di $12^{\circ} \mathrm{C}$ ed una precipitazione annua media di circa $790 \mathrm{~mm}$.

L'area è situata su una formazione geologica formata da scisti argillosi, policromi, scagliettati e, nella parte superficiale, interessata da fenomeni franosi. Dal punto di vista pedologico, il suolo rientra nel tipo "vertic ustorthens", secondo la classificazione USDA. Altre proprietà importanti sono rappresentate da una modesta permeabilità e dai caratteri vertici del terreno argilloso, ben apprezzabili durante il periodo estivo per le evidenti crepacciature.

I dettagli sulla strumentazione di misura e sulla di- 


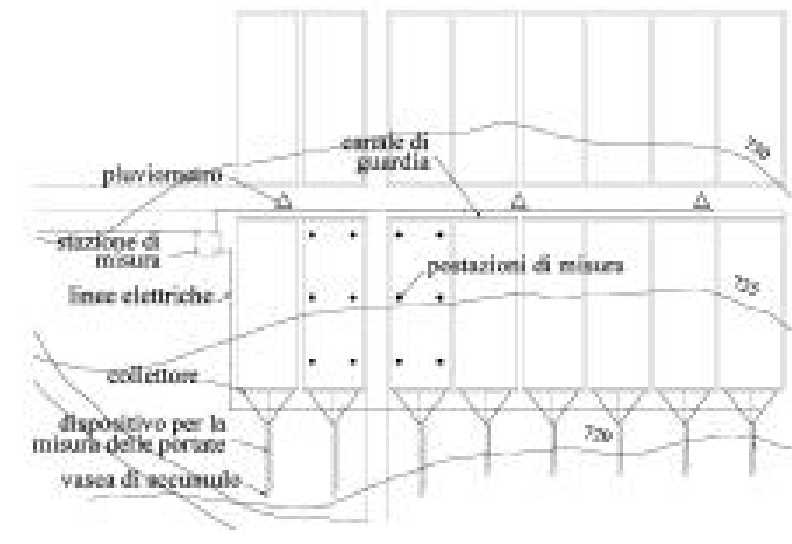

Fig. 1 - Planimetria del campo di Guardia Perticara.

Fig. 1 - Map of the Guardia Perticara site.

sposizione delle parcelle sperimentali sono riportati in Ciollaro et al. [2].

Con riferimento all'indagine oggetto del presente studio, le prove hanno interessato due parcelle sulle quali sono state approntate 6 postazioni di misura dotate di un tubo di accesso per la sonda a neutroni, tre sonde TDR e due tensiometri. Il tubo in alluminio per l'accesso della sonda a neutroni è stato infisso nel terreno per una profondità di $1,5 \mathrm{~m}$. Con frequenza settimanale sono state effettuate le misure a sei diverse profondità, nel campo $20 \div 110 \mathrm{~cm}$. I tensiometri a mercurio sono stati posizionati rispettivamente ad una profondità pari a $80 \mathrm{~cm}$ e $100 \mathrm{~cm}$. Le letture sono state eseguite ogni tre giorni. Le postazioni sono state completate con tre sonde TDR per la misura in automatico a tre diverse profondità. La sonda più superficiale è stata infissa verticalmente $0 \mathrm{~cm}$ e $15 \mathrm{~cm}$, le altre due sono invece state posizionate orizzontalmente alle profondità di $30 \mathrm{~cm}$ e $60 \mathrm{~cm}$.

Nella figura 2 sono mostrati gli andamenti nel tempo, relativi ad un anno di osservazioni, delle piogge e dei contenuti idrici misurati con le sonde TDR e con la sonda a neutroni

La frequenza di acquisizione con le sonde TDR ha consentito di descrivere con elevata risoluzione temporale l'andamento dei contenuti d'acqua, rispetto alla sonda a neutroni che ha tuttavia consentito misure di maggiore dettaglio lungo il profilo di suolo

Le misure prese in esame in questo studio sono relative al periodo $12-26$ aprile.

\subsection{LA CARATTERIZZAZIONE IDRAULICA DEI SUOLI}

\subsection{Soil Characterization}

Il suolo è stato caratterizzato idraulicamente attraverso il prelievo di campioni non rimaneggiati a tre diverse profondità in dieci siti. Sono stati utilizzati campionatori di diametro interno pari ad $8,0 \mathrm{~cm}$ ed altezza pari a $12,0 \mathrm{~cm}$. In laboratorio, attraverso prove di evaporazione dei campioni sono state ottenute le curve di ritenzione idrica e di conducibilità secondo la metodologia proposta da Ciollaro e Comegna [3]. Le curve così ottenute sono state interpolate con l'espressione analitica proposta da van Genuchten, ricavandone il valore dei relativi parametri. Per ciascuna profondità indagata le curve medie caratteristiche non hanno mostrato significative differenze fra i diversi siti [10].

Per la dimensione dei campioni e per il marcato stato di aggregazione del suolo, si è ritenuto che le funzioni idrauliche fornite dalle indagini sperimentali fossero rappresentative della sola componente matriciale del mezzo poroso.

Le informazioni corrispondenti alla parte strutturale del suolo sono invece state ricavate attraverso prove condotte su grandi colonne di suolo (diametro 30,5 $\mathrm{cm}$ ed altezza $60,0 \mathrm{~cm}$ ) prelevate in tre diverse postazioni nelle parcelle sperimentali.

I campioni, superiormente sigillati lungo la linea di contatto con il contenitore, sono stati sottoposti ad un processo di infiltrazione con l'ausilio di un simulatore di pioggia costituito da un disco in perspex di diametro $30,0 \mathrm{~cm}$, munito di 164 aghi per assicurare una distribuzione uniforme della pioggia.

I volumi in entrata, quelli drenati e quelli corrispondenti ad un eventuale deflusso sono stati rilevati a tempi prefissati attraverso tre celle di carico collegate ad un sistema di acquisizione automatica.

I valori del contenuto idrico iniziale e delle sue variazioni durante il processo di inumidimento sono stati rilevati con l'utilizzo di cinque sonde TDR, poste orizzontalmente ad intervalli di $10,0 \mathrm{~cm}$ dalla superficie del suolo, a partire da una profondità di $5,0 \mathrm{~cm}$.

In laboratorio le colonne sono state poggiate su un fondo drenante realizzato stratificando materiali a diversa granulometria, che ha consentito di quantificare, separandoli, anche gli eventuali flussi lungo la parete interna della colonna da portare in conto in fase di elaborazione dei dati.

Il primo processo di infiltrazione ha avuto inizio a partire da condizioni iniziali medie di umidità pari all' $15 \%$, con un valore dell' $11 \%$ sulla sommità del campione e del $18 \%$ in mezzeria. L'intensità di pioggia applicata è stata di $14 \mathrm{~mm} / \mathrm{h}$. L'apporto è stato interrotto quando non sono state più osservate variazioni di contenuto idrico. Il deflusso è iniziato dopo solo 90 minuti, mentre nella parte inferiore del campione non si registravano variazioni di contenuto d'acqua. Di conseguenza si può ritenere che il deflusso sia stato originato dal trasporto nei macropori.

Le prove successive sono state eseguite a partire da contenuti d'acqua iniziali variabili fra il $30 \%$ ed il $41 \%$, mentre è stata incrementata l'intensità di pioggia fino a valori dell'ordine di $42 \mathrm{~mm} / \mathrm{h}$.

Durante tutti gli esperimenti, in condizioni di suolo umido, è stato sempre osservato un trasporto attraverso i macropori anche quando non erano visibili fratture. Non è stato mai osservato un accumulo di acqua sulla superficie del suolo, anche se l'intensità di pioggia era maggiore della massima velocità di infiltrazione propria della matrice solida.

In figura 3 sono riportate le curve caratteristiche 


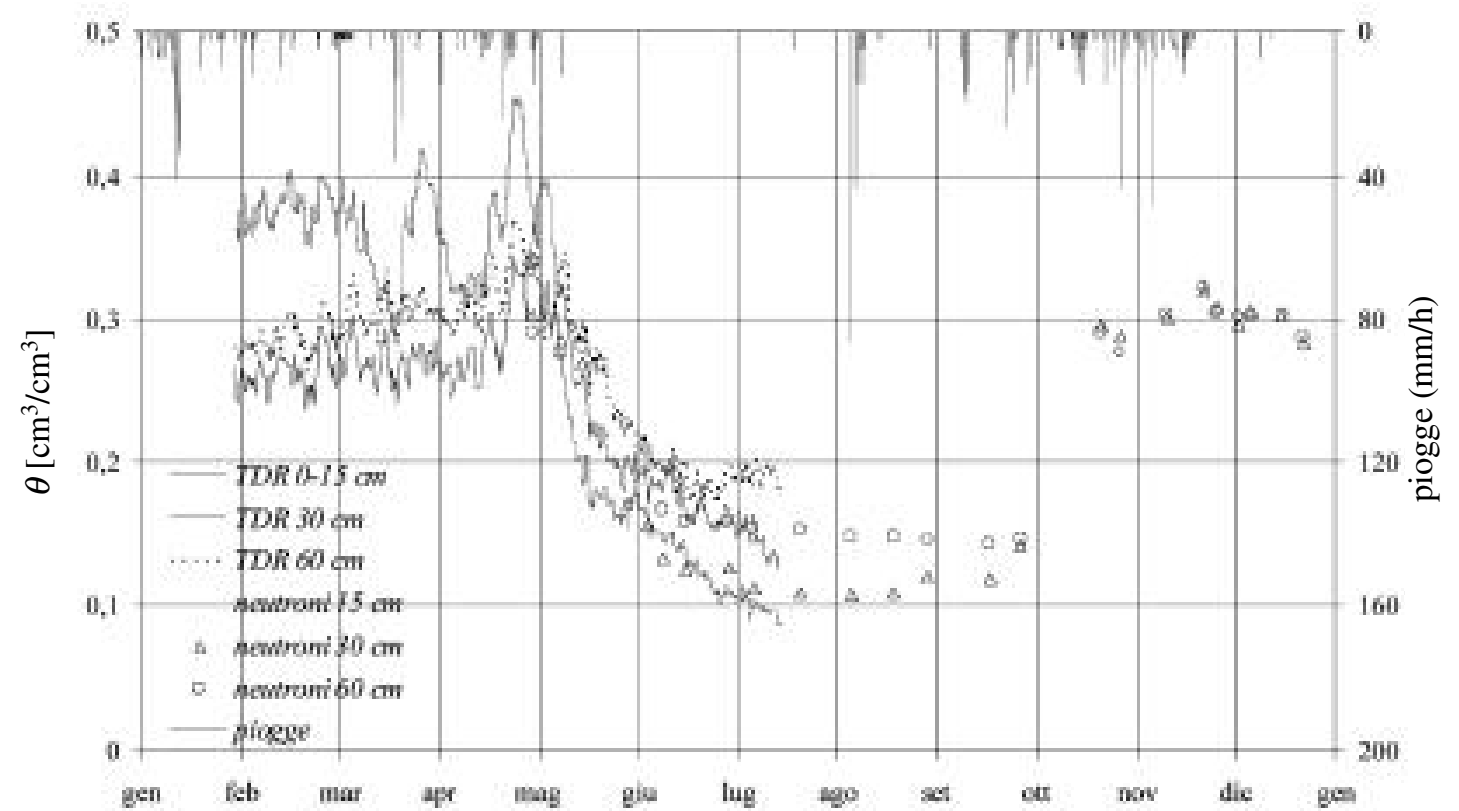

Fig. 2 - Intensità delle precipitazioni ed evoluzione spazio-temporale dei contenuti d'acqua misurati con sonde TDR (linee) e sonda a neutroni (simboli).

Fig. 2 - Rain intensity and spatio-temporal evolution of water content as measured by TDR (lines) and neutron probe (symbols).
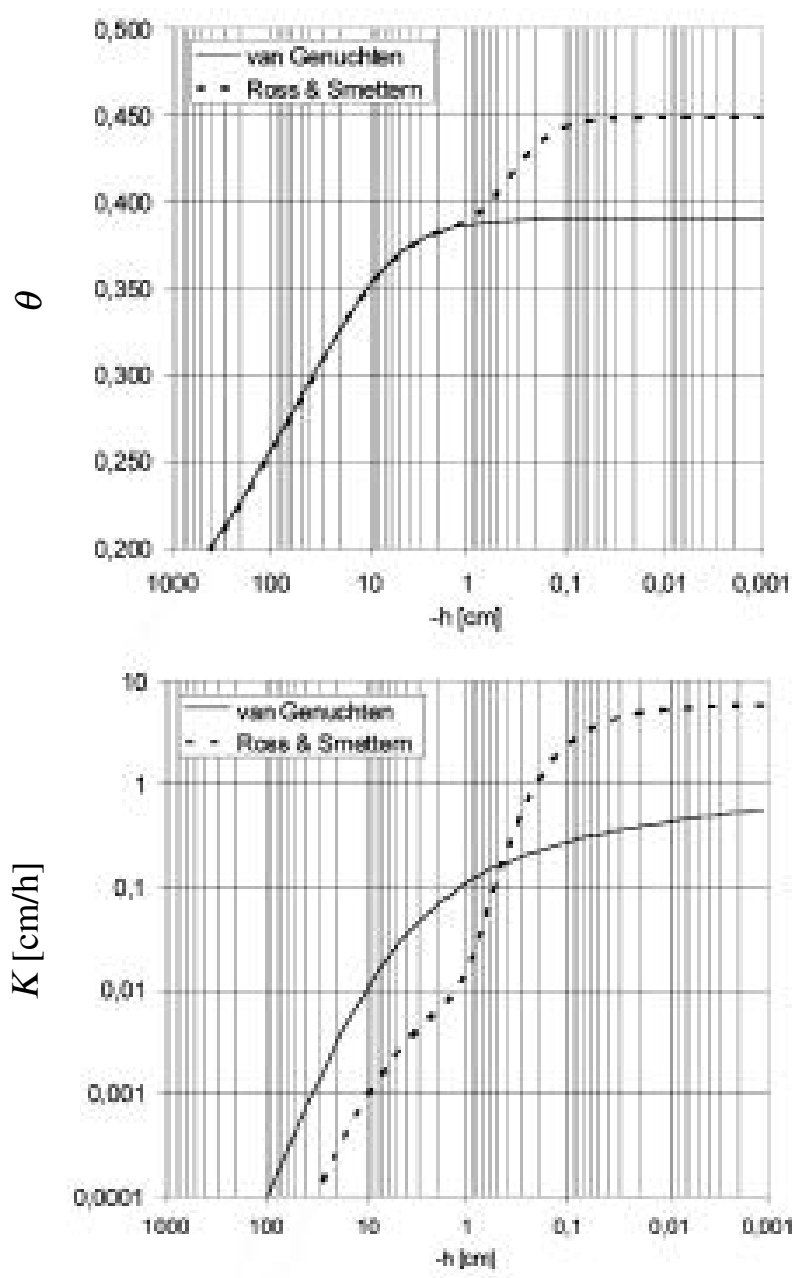

Fig. 3 - Caratteristiche idrauliche medie del suolo.

Fig. 3 - Average soil hydraulic properties. del suolo, ottenute sovrapponendo le caratterizzazioni effettuate sui campioni e sulle colonne, avendo assunto che la parte matriciale della ritenzione idrica relativa alle colonne corrispondesse alla curva di ritenzione ottenuta sui campioni. Alle due curve di ritenzione riportate in figura corrispondono due differenti stime della curva di conducibilità ottenute utilizzando il modello di Mualem, anche in dipendenza del più elevato valore della conducibilità per $h=0$ misurato sulle colonne.

\section{RISULTATI E CONCLUSIONI}

\section{Results And Conclusions}

Nei grafici riportati in figura 4 sono rappresentate le intensità di pioggia, i valori di contenuto d'acqua misurati con le sonde TDR e quelli simulati per tre diverse profondità.

Nel primo grafico le simulazioni sono state effettuate risolvendo la (1) nell'ipotesi di un profilo non stratificato ed assumendo che le proprietà idrauliche, rappresentative del mezzo allo studio, fossero quelle determinate sui campioni di suolo. Le curva di ritenzione idrica e di conducibilità idraulica erano perciò quelle identificate come "van Genuchten" nella figura 3 . In questa configurazione, le simulazioni consentono di descrivere correttamente l'andamento del contenuto d'acqua nel tempo solo alla prima profondità, mentre insoddisfacenti risultano essere le simulazioni relative alle altre due profondità. In particolare, si noti come le simulazioni forniscano una rappresentazione non corrispondente alla reale evoluzione dei profili 
misurati di contenuto d'acqua, che mostrano un suolo più umido $\mathrm{a} 60 \mathrm{~cm}$ e più secco a $30 \mathrm{~cm}$ di profondità.

Le simulazioni riportate nel secondo grafico si riferiscono invece ad una formulazione bimodale delle proprietà idrauliche rappresentate questa volta dalle curve identificate come "Ross \& Smettem" nella figura 3. In questa configurazione, le simulazioni, pur riproducendo l'inversione osservata alle profondità di 30 e $60 \mathrm{~cm}$, forniscono ancora una rappresentazione non realistica dell'evoluzione dei profili di contenuto d'acqua, con una evidente sottostima dell'umidità in superficie, per effetto del maggiore drenaggio riconducibile alla particolare formulazione delle funzioni idrauliche.

Si deve comunque considerare che il suolo oggetto dell'indagine viene periodicamente sottoposto a lavorazioni superficiali che interessano i primi $20 \mathrm{~cm}$ del profilo che, per quanto osservato nell'introduzione, inducono una disaggregazione nello strato lavorato, con una conseguente modifica delle caratteristiche
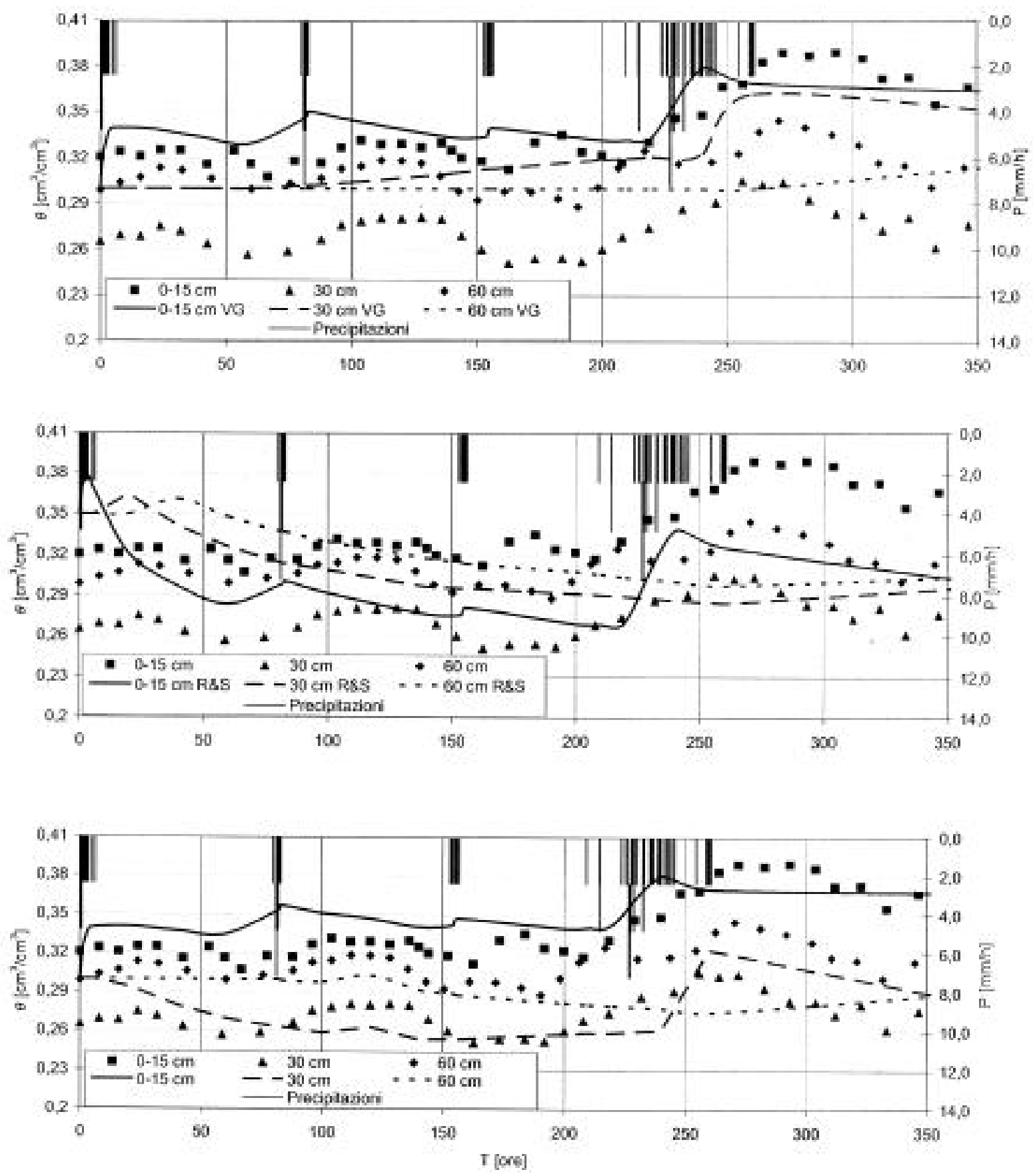

Fig. 4 - Confronto fra andamenti di contenuto d'acqua nel tempo, a tre profondità diverse, misurati e simulati; il primo grafico riporta le simulazioni con suolo caratterizzato con le funzioni di van Genuchten, il secondo con Ross e Smettem, il terzo è stato ottenuto considerando la stratificazione indotta dalle lavorazioni

Fig. 4 - Comparison of measured and simulated water content evolution at three different depth.;Tthe first and the second graphs refer to numerical simulations based on van Genuchten and Ross\&Smettem water retention, respectively, while the third graph refer to the case of layering induced by tillage. 

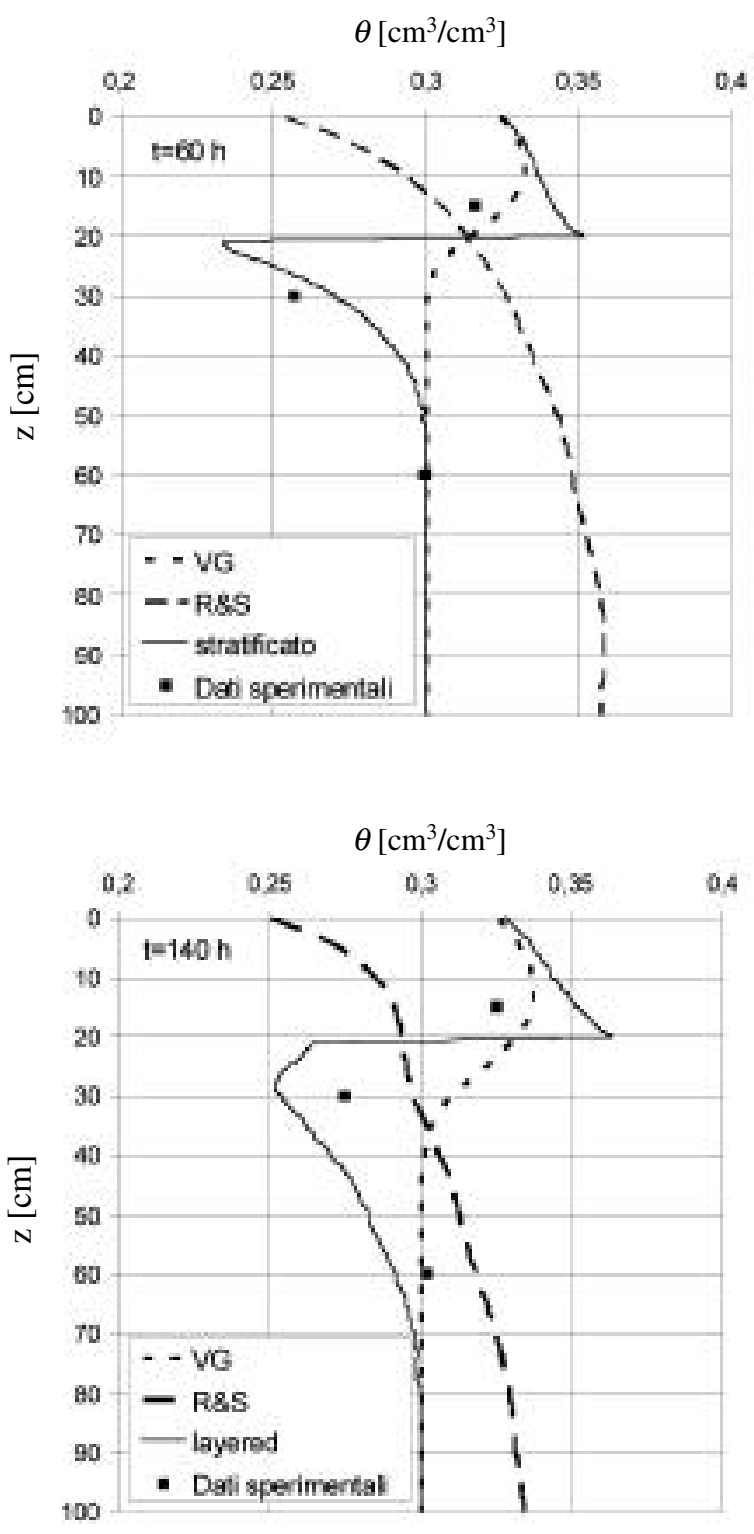

Fig. 5 - Confronto fra profili di umidità misurati e quelli simulati con i tre diversi approcci e relativi a due tempi diversi.

Fig. 5 - Comparison of soil water content profiles as measured and simulated by the three different approaches at two different times.

idrauliche del suolo, con effetti che permangono nel mese di aprile (periodo a cui i dati si riferiscono).

È apparso perciò lecito assumere il profilo di suolo come costituito da almeno due strati, il primo, dello spessore di $20 \mathrm{~cm}$, generato dalle lavorazioni, e caratterizzato da proprietà idrauliche del tipo van Genuchten, il secondo rimasto inalterato, caratterizzato da proprietà del tipo Ross \& Smettem.

I risultati delle simulazioni condotte in questa configurazione sono riportati nel terzo grafico della figura 4 . Come si può notare il modello fornisce stime migliori, almeno per le prime 200 ore. Non spiegato rimane invece l'incremento dei contenuti d'acqua osservato alla profondità di $60 \mathrm{~cm}$ a partire dalle 200 ore. Questo particolare comportamento può essere ragionevolmente spiegato in considerazione degli eleva- ti valori di contenuto d'acqua registrati in superficie nel periodo esaminato, che determinano presumibilmente la saturazione della matrice e la conseguente attivazione di un flusso nei macropori non capillari, non descrivibile in modo adeguato mediante la classica equazione di Richards.

In generale, si ritiene comunque che la formulazione adottata fornisca una rappresentazione molto prossima alla realtà dei profili di umidità, come confermato nella figura 5 , che mostra un confronto, per due tempi diversi di simulazione, fra le misure e le simulazioni riferite alle tre diverse configurazioni utilizzate in questo lavoro.

I risultati evidenziano che quando gli effetti delle eterogeneità a scala locale vengono esplicitamente considerati, si ottengono predizioni soddisfacenti dei processi osservati. Tuttavia, perché questi approcci possano essere efficacemente applicati, è necessario che il comportamento idrologico del suolo in prossimità della saturazione venga accuratamente caratterizzato attraverso dettagliate descrizioni sperimentali delle funzioni idrauliche. Ciò soprattutto in considerazione dell'importante impatto che i vuoti strutturali possono avere sulla rapidità delle dinamiche di infiltrazione e di drenaggio che possono attivarsi in dipendenza delle quantità d'acqua somministrate in superficie e dei contenuti d'acqua iniziali lungo il profilo.

È nostra convinzione che l'adozione di un approccio che assuma la continuità del mezzo poroso strutturato, sebbene rappresenti in alcuni casi un'approssimazione non strettamente applicabile per mezzi porosi fratturati, possa risultare comunque adeguata. Si ritiene, in definitiva, che, date le incertezze nella completa interpretazione dei processi di flusso preferenziale, l'equazione di Richards, seppure formalmente non sempre valida, possa ancora fornire un'utile descrizione qualitativa degli stessi processi.

\section{Bibliografia}

\section{References}

[1] Beven K., Germann P., Macropores and Water Flow in Soils. Water Resources Research 18, 1311-1325, 1982.

[2] Ciollaro G., Coppola A., Damiani P., Sommella A., Rilievo di grandezze idro-meteorologiche per la stesura di un bilancio idrico alla scala parcellare. Idronomia Montana, n. 21, 2002.

[3] Ciollaro G., Comegna V., Methodology for the study of hydraulic properties of soil on a plot scale. Atti $11^{\circ}$ International Congress on Agricultural Engineering. Dublino, 4-8 settembre 1989. pp. 471-480, 1989.

[4] DURNER W., Hydraulic conductivity estimation for soils with heterogeneous pore structure. Water Resour. Res. 30:211-223, 1994.

[5] Horn R., Taubner H., WuttKe M., Baumgartl T., Soil physical properties related to soil structure. Soil Tillage Res., 30, 187-216, 1994. 
[6] JuRY W., Roth, K., Evaluating the role of preferential flow on solute transport through unsaturated field soils. In: K. Roth, H. Flühler, W.A. Jury and J.C. Parker (Editors), Field scale water and solute flux in soils. Birkhäuser Verlag, Basel-Boston-Berlin, pp. 23-28, 1990.

[7] Kutilek M., Nielsen D.R., Soil Hydrology. Catena Verlag, Germany. pp. 97-98, Darcian e Non-Darcian Flow, 1994

[8] Mualem Y., A new model for predicting the hydraulic conductivity of unsaturated porous media. Water Resour. Res. 12:513-522, 1976.

[9] Ross P.J., Smettem R.J., Describing soil hydraulic properties with sums of simple functions. Soil Sci. Soc. Am. J. 57:26-29, 1993.

[10] Santini A., Romano N., Ciollaro G., Comegna V., Evaluation of a laboratory inverse method for determining unsaturated hydraulic properties of a soil under different tillage practices. Soil Science, 160: 340$351,1995$.

[11] Soil Science Society of America, Glossary of soil science terms 1996. Soil Sci. Soc. Am., Madison, Wis., 1997.

[12] van Genuchten M. Th. and Nielsen D.R., On describing and predicting the hydraulic properties of unsaturated soils. Ann. Geophis. 3: 615-628, 1985.

Parole chiave: modelli uni-bimodali di ritenzione idrica, bilancio idrologico, metodo TDR e neutronico.

\section{SUMMARY}

\section{EFFECT OF TILLAGE LAYERING ON THE HYDROLOGICAL BEHAVIOR OF A STRUC- TURED SOIL}

Soils often exhibit a variety of small-scale heterogeneities such as cracks, inter-aggregate macropores and voids which partition flow into separate regions. In this paper an approach is discussed for characterizing the hydrological behavior of heterogeneous soils, in the presence of structural inter-aggregate macropores or even shrinkage cracks. Accordingly, special emphasis is given to the effect of micro-macro-heterogeneity and soil structure on water flow processes at local scale. The discussion is limited to a mechanistic approach, known to be based on physical concepts and laws. Theoretical discussion and experimental evidence of the structural effects on the hydrological behavior of soils are provided. Flexible retention relations in describing the retention data of aggregated soils are evaluated, with the principal aim of assessing their predictive capability for estimating the hydraulic conductivity function and for describing flow in a composite porosity framework. Their predictive capability is independently tested by using a set of water content observations at different depths. The results enable a detailed discussion on model parameterization and related parameter uncertainty to be made.

Key words: uni/bimodal water retention model, idrological balance, TDR and Neutron probe methods. 
006_Damiani(504)_45 30-07-2008 10:14 Pagina 52

$\phi$

$\phi$ 